\title{
Towards Giga-pixel Microscopy
}

\author{
Guoan Zheng, Xiaoze Ou and Changhuei Yang \\ Department of Electrical Engineering, California Institute of Technology, Pasadena, CA \\ 91125, USA \\ gazheng@,caltech.edu
}

\begin{abstract}
We report a wide field-of-view (FOV) microscopy imaging system that is capable to capture a $10 \mathrm{~mm} * 7.5 \mathrm{~mm}$ FOV image with submicron resolution, resulting in 0.54 giga-pixels across the entire image.

OCIS codes: (170.0110) Imaging system; (170.4730) Optical pathology.
\end{abstract}

\section{Introduction}

Low-cost, high-resolution and wide field-of-view (FOV) microscopy imaging system is highly sought for biomedical and pharmaceutical applications, such as digital pathology diagnostics, cancel cell detection and drug screening. However, due to the limited FOV of the conventional microscope objective lens, large regions of a sample cannot be imaged in high resolution with one camera exposure. For example, the resolution of a conventional $20 \mathrm{X}$ objective lens ( 0.4 numerical aperture) is about $0.7 \mu \mathrm{m}$ and the FOV is less than $1 \mathrm{~mm}$ in diameter. The resulting space-bandwidth-product (SBP) is about 8 mega-pixels (the number of independent pixels to characterize the captured image). One can use other objective lenses with different magnifications, while the SBP remains the same.

In the past years, exciting progresses have been made in wide FOV imaging system, including digital in-line holography [1], focus-grid scanning illumination [2, 3], off-axis holography microscopy [4, 5] and adaptive scanning technique [6]. However, there are also some limitations associated with these approaches. For example, digital in-line holography only works well with the sparse objects with true boundary constrain. Dense sample, such as a pathology slide, is challenging for this approach due to the significant distortion of the wave transmitted through the sample. In the case of focus-grid scanning microscopy, the sample is illuminated with a grid of focused light spots (by the holography effect or the Talbot effect), and the transmission of the light spots through the sample is projected onto an imaging sensor. The optical efficiency for generating such a focused-grid is relatively low and the resolution is limited by the spot size. Furthermore, both approaches are based on light interference, and thus, the coherence-based noise sources would be present and would need to be addressed. Recently, we demonstrated an onchip wide FOV microscopy solution to address the cell culture needs, termed ePetri dish [7]. Such an approach is based on incoherent light illumination and it can generate a $\sim 0.25$ giga-pixel image (effective pixel by Nyquist rate) using a commercial available 5 mega-pixel image sensor. To complement this on-chip microscopy solution, in this paper, we demonstrate a low-cost giga-pixel microscopy imaging system which can be operated off-chip; therefore, we can address the need for high throughput microscopy imaging in boarder biomedical, pharmaceutical and forensic applications.

\section{The prototype setup of the giga-pixel microscopy system}

The configuration of this imaging system is based on two simple items only: a low-cost consumer flatbed scanner and a commercial available closed-circuit-television (CCTV) lens.

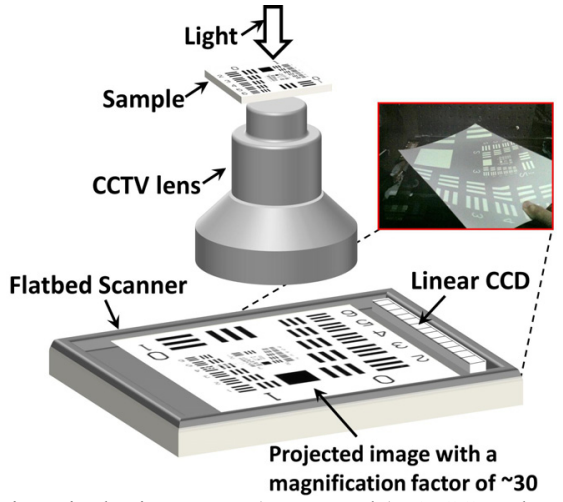

Figure 1. The setup of the proposed giga-pixel microscopy (not to scale). A CCTV lens is used to magnify the sample by a factor of 30 and a scanner is used to capture the projected image. 
In our setup (Fig. 1), we put our sample at the image plane to replace the CMOS/CCD imager and use the CCTV lens to magnify sample, i.e. using the lens in the reverse manner. With a magnification factor of $\sim 30$, we then scan the projected image using a consumer flatbed scanner s. We show that, such a system is capable to capture a 0.54 giga-pixel microscopy image with a FOV of $10 \mathrm{~mm} * 7.5 \mathrm{~mm}$ and the sub-micron resolution is achieved across the entire FOV.

\section{Results}

For application demonstration, we acquired an image of a pathology slide (human metastatic carcinoma to liver, Carolina Biological Supply) in Fig. 2. We used R/G/B LED light sources for three color illuminations. The sample slide is manually tuned to its in-focus position using a mechanical stage. Three images (for R/G/B) are separately acquired, normalized, aligned and then combined into the final color image. The FOV is about $10 \mathrm{~mm} * 7.5 \mathrm{~mm}$, containing 26400 pixels * 20400 pixels. Fig.2 (b1), (b2) and (c1), (c2) are the corresponding expanded view for Fig. 2(a). We can see that our system can render a wide FOV image and still provide fairly good resolution. We also note that other imaging functionaries such as 3D, darkfield [8] and phase imaging can also be incorporated into the proposed wide FOV platform.

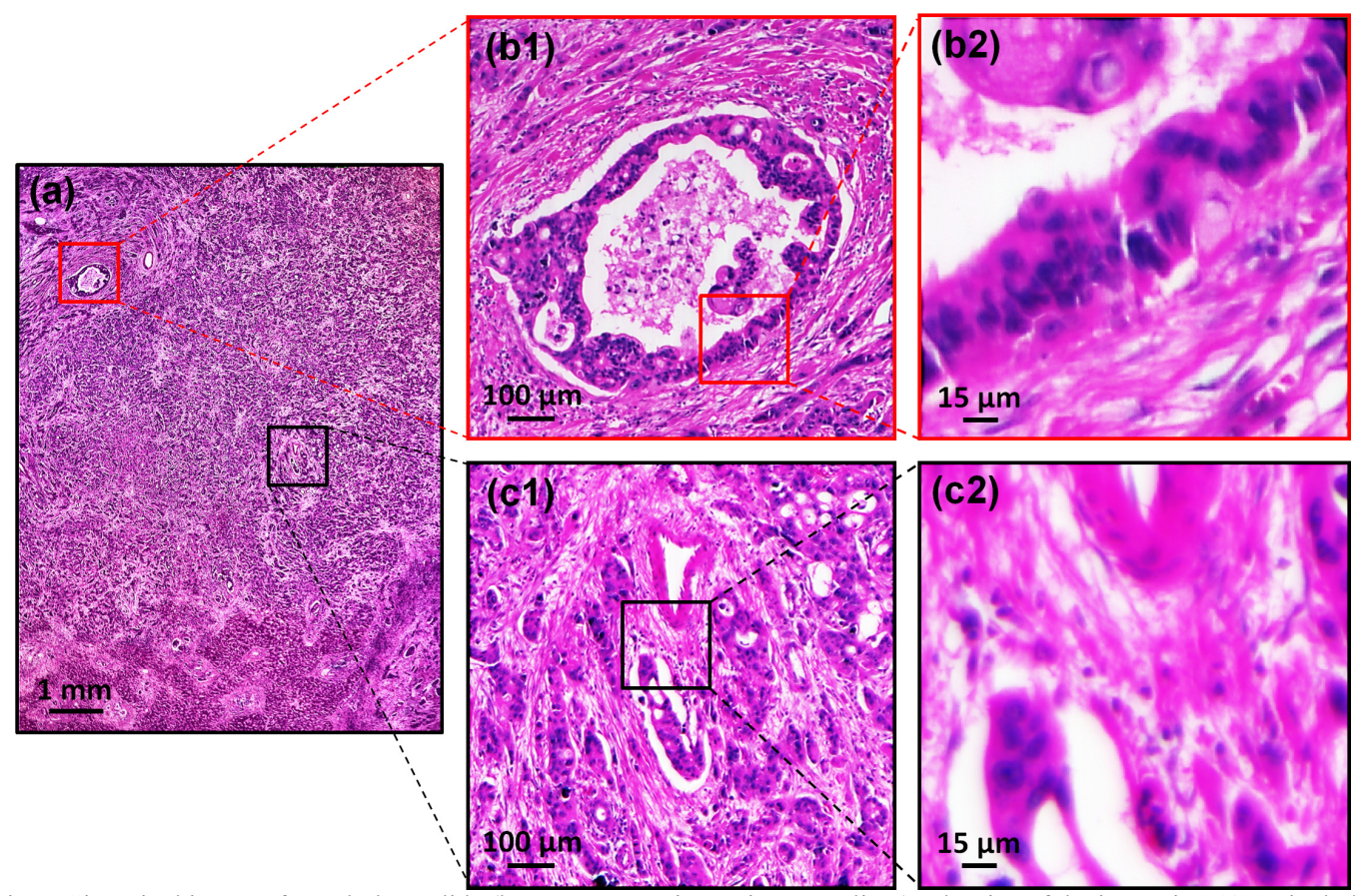

Fig. 2 Giga-pixel image of a pathology slide (human metastatic carcinoma to liver). The size of the image is 26400 pixels * 20400 pixels, and the FOV is about $10 \mathrm{~mm} * 7.5 \mathrm{~mm}$. (a) The full frame of the captured image. (b1), (b2) and (c1), (c2) are the expanded view of the (a).

\section{References}

[1] W. Bishara, T. Su, A. Coskun, and A. Ozcan, "Lensfree on-chip microscopy over a wide field-of-view using pixel super-resolution," Optics Express 18, 11181-11191 (2010).

[2] J. Wu, X. Cui, G. Zheng, Y. M. Wang, L. M. Lee, and C. Yang, "Wide field-of-view microscope based on holographic focus grid illumination," Optics letters 35, 2188-2190 (2010).

[3] J. Wu, G. Zheng, Z. Li, and C. Yang, "Focal plane tuning in wide-field-of-view microscope with Talbot pattern illumination," Optics letters 36, 2179-2181 (2011).

[4] J. Di, J. Zhao, H. Jiang, P. Zhang, Q. Fan, and W. Sun, "High resolution digital holographic microscopy with a wide field of view based on a synthetic aperture technique and use of linear CCD scanning," Applied optics 47, 5654-5659 (2008).

[5] M. Lee, O. Yaglidere, and A. Ozcan, "Field-portable reflection and transmission microscopy based on lensless holography," Biomedical Optics Express 2, 2721-2730 (2011).

[6] B. Potsaid, F. P. Finger, and J. T. Wen, "Automation of challenging spatial-temporal biomedical observations with the adaptive scanning optical microscope (ASOM)," Automation Science and Engineering, IEEE Transactions on 6, 525-535 (2009).

[7] G. Zheng, S. A. Lee, Y. Antebi, M. B. Elowitz, and C. Yang, "The ePetri dish, an on-chip cell imaging platform based on subpixel perspective sweeping microscopy (SPSM)," Proceedings of the National Academy of Sciences 108, 16889-16894 (2011).

[8] G. Zheng, C. Kolner, and C. Yang, "Microscopy refocusing and dark-field imaging by using a simple LED array," Optics letters 36, $3987-$ 3989 (2011). 